\section{Identification of the Main Ethylene- diamine Condensate of Noradrenaline with that of Catechol}

My attention has been directed to a recent com munication ${ }^{1}$ by T. Nagatsu and K. Yagi dealing with the identification of the main ethylenediamine condensate of noradrenaline with that of catechol. The authors report some experiments, quote an infra-red spectrum and two microphotographs of crystals. Also, the sentence occurs: "However, the precise identification of these compounds in the pure form has not yet been reported".

In fact, not only "the precise identification of these compounds", but also a rigorous proof of the chemical structure and an independent synthosis of those compounds was reported in a detailed paper by J. HarleyMason and A. H. Laird more than two years ago ${ }^{2}$, and all the experiments reported by the Japanese authors are contained in this paper.

\section{J. HARLEX-MASON}

University Chomical Laboratory, Lensfield Road, Cambridge.

1 Nature, 198, 484 (1962).

${ }^{2}$ Tetrahedron, 7, 70 (1959)

Though we missed the report by J. Harley-Mason and A. H. Laird (Tetrahedron, 7, 70; 1959), we realize that the results reported in our communication are

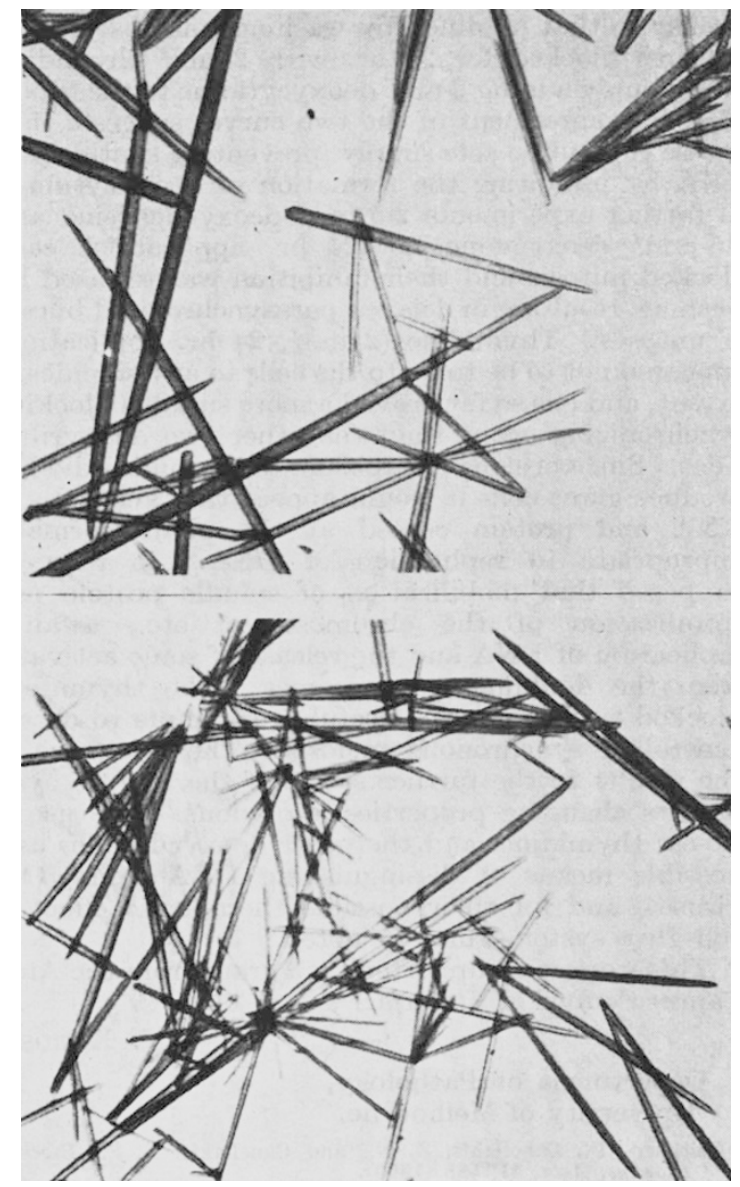

Fig. 1. Picrate crystal of a main ethylenediamine condensate of catechol (above); noradrenaline (below) $(x, 1,000)$ identical with a part of the results reported by them. Therefore, as a result, our communication serves to confirm their results.

We may add another fact confirming the identification of both the main condensates. We isolated both substances as picrates. Both picrates formed similar, needle-shaped, orange red crystals (Fig. 1).

The infra-red spectra of both picrates were identical. The result of elementary analysis of picrate crystals of the main noradrenaline-ethylenediamine condensate was as follows: eale. for $\mathrm{C}_{10} \mathrm{H}_{10} \mathrm{~N}_{4} \cdot 2 \mathrm{C}_{6} \mathrm{H}_{3} \mathrm{~N}_{3}$ (). $\mathrm{C}: 41 \cdot 00, \mathrm{H}: 2 \cdot 500, \mathrm{~N}: 21 \cdot 74, \mathrm{O}: 34 \cdot 76$; found, $\mathrm{C}$ : $40 \cdot 96, \mathrm{H}: 2 \cdot 695, \mathrm{~N}: 2 \mathrm{~J} \cdot 67, \mathrm{O}: 34 \cdot 55$ per cent.

This result corresponds with the dipicrate of tetrahydro-tetra-aza-anthracene, confirming tho result of Harley-Mason and Laird.

Toshihare Nagatsu

Department of Biochemistry,

School of Medicine,

Nagoya University, Nagoya, Japan.

\section{Determination of Free Amino-Acids in Human Ejaculate by Ion Exchange Chromatography}

Reports on investigations concerning the content of free amino-acids in human ejaculates are rare. Sakar, Luecke and Duncan ${ }^{1}$ estimated the aminoacid composition by microbiological methods. Adam and Korting ${ }^{2}$, Jacobsson ${ }^{3}$ and Lundquist ${ }^{4}$ separated amino-acids from human sperm liquor qualitatively by paper chromatography. Keutel and Gabsch used paper chromatography for qualitative and quantitative determinations of amino-acids from ejaculates ${ }^{5}$.

We analysed quantitatively the content of free amino-acids in sperm liquor by ion exchange chromatography ${ }^{6}$ using an automatic recording equipmont ${ }^{7}$. The method is capable of giving reproducible recov. eries of better than $100 \pm 3$ per cent for quantities ranging from 0.25 to 2 umoles of amino-acid. One complete analysis required 2 days.

Four to six hours after ejaculation, a 10 -fold amount of 1 per cent picric acid according to Hamilton's method ${ }^{8}$ was added to the ejaculate. The picric acid precipitate was removed promptly by centrifugation. To remove excess picric acid from the protein-free ejaculate, the solutions were passed through columns of 'Dowex 2' (2 cm. diam. $)^{9}$; a bed $2 \mathrm{~cm}$. in height was used. Tho colourless effluents were filtered with 'Celite' if cloudy and hydrolysed for $24 \mathrm{hr}$. with $100 \mathrm{ml}$. $6 \mathrm{~N}$ hydrochloric acid under reflux to split peptides and conjugated amino-acids, if present. The hydrolysate was dehydrated three times in a rotary evaporator, treated with 'Celite', when necessary and made up to a volume of 5-10 ml. with a $0.2 N$ sodium citrate buffer, $p H \quad 2.2{ }^{10}$. All solutions were kept frozen prior to chromatography. An amount of the hydrolysato corresponding to $0.5 \mathrm{ml}$. of the original ejaculate was chromatographed in most instances. The samples were analysed on columns of 'Amberlite $I R$-120' using a solf-designed amino-acid analyser"1.

Table 1 shows the average values of 10 patients with normospermia, 5 with azoospormia combined with Leydig cell insufficiency and one patient with asper. mia due to deformity of accessory sexual glands. Fig. 1 demonstrates a typical effluent curve of free amino-acids from an ejaculate of a case of normospermia. 